\title{
Oclusão traqueal para fetos com hérnia diafragmática congênita: uma revisão integrativa
}

Tracheal occlusion for fetus with congenital diaphragmatic hernia: integrative review

Oclusión traqueal para fetos hernia diafragmática congénita: una revisión integrativa

Laís Mesquita Mororó Aragão ${ }^{1 *}$, Maria Carolina Oliveira Azevedoㄹ, Paula Sabrina Martins Barros", Tereza Cristina de Carvalho Souza Garcês ${ }^{1}$, Priscila Favoritto Lopes ${ }^{1}$.

\section{RESUMO}

Objetivo: Analisar se o tempo de diagnóstico da Hérnia Diafragmática Congênita (HDC) interfere na elegibilidade para a técnica e consequentemente leva a casos mais graves que não iriam sobreviver. Métodos: Realizou-se uma revisão integrativa de literatura, com abordagem qualitativa, cujas bases de dados consultadas foram LILACS e PubMed, com os descritores: "Congenital Diaphragmatic Hernia", "Surgical treatment", "Surgical procedure" e "Operative", entre os anos de 2012 e 2018. Resultados e discussão: Identificaram-se 687 artigos e 12 artigos foram elegíveis para a análise. Observou-se, que cinco artigos relacionavam a idade de diagnóstico gestacional com a mortalidade e morbidade. Com os dados obtidos, foram encontrados que até o momento há disparidade entre os resultados sobre a técnica FETO e que a idade de diagnóstico é um fator preditivo isolado de prognóstico. Conclusão: A HDC diagnosticada no pré-natal, ainda permanece um desafio, pois, mesmo nos casos mais graves detectados precocemente, é necessário que o serviço tenha estrutura para realizar o atendimento adequado. Espera-se que este estudo possa contribuir para o campo de conhecimento da cirurgia pediátrica, abrindo caminhos para novas pesquisas.

Palavras-chave: Hérnias Diafragmáticas Congênitas, Idade Gestacional, Análise de sobrevida.

\begin{abstract}
Objective: Analyzing the time of the Congenital Diaphragmatic Hernia diagnostic interferes in the eligibility for technique and consequently leads to more severe cases they wouldn't survive. Methods: It performed an integrative literature review, with qualitative approach, whose consulted data bases were SciELO and PubMed, with the descriptors: "Congenital Diaphragmatic hernia", "surgical treatment", "surgical procedure" and "operative", between 2012 and 2018. Results: There were identified 687 articles and 12 articles were eligible for analysis. They observed that five articles relating to the gestation age diagnostic with mortality and morbidity. With the data found, until now, there's a mismatch between the results of FETO and that the age of diagnosis is an isolated predictive prognosis factor. Conclusion and discussion: The early diagnosis of HDC remains a challenge, since more severe cases are detected precociously and that not every service is able to offering the proper treatment for this pathology, whether it's from lack of structure, material or absence of centers that perform FETO. It's expected that this study can contribute to the field of pediatric surgery, opening way to new research.
\end{abstract}

Key words: Congenital Diaphragmatic Hernia, Gestacional Age, Survival Analysis.

${ }^{1}$ Discente do curso de Medicina da Faculdade de Ciências Humanas, Exatas e da Saúde do Piauí (FAHESP/IESVAP), Parnaíba-Piauí. *E-mail: lais_mororo@hotmail.com 


\section{RESUMEN}

Objetivo: Analizar si el tiempo de diagnóstico de la HDC interfiere en la elegibilidad para la técnica y consecuentemente conduce a casos más graves que no sobrevivan. Metodos: Se realizó una revisión integrativa da literatura, con abordaje cualitativa, cuyas bases de datos consultadas fuerón SciELO y PubMed, con los descriptores: "Congenital Diaphragmatic hernia", "Surgical treatment", "Surgical procedure" y "Operative", entre los años 2012 y 2018. Resultados: Se identificaron 687 artículos y 12 artículos fueron elegibles para el análisis. Se observó que cinco artículos relacionaban la edad de diagnóstico gestacional con la mortalidad y morbilidad. Con los datos obtenidos, se encontrarón que hasta el momento hay disparidad entre los resultados sobre la FETO y que la edad de diagnóstico es un factor predictivo aislado de pronóstico. Consideraciones finales: La HDC diagnosticada prenatalmente sigue siendo un desafío, porque incluso en los casos más graves detectados en forma temprana, es necesario que el servicio tenga la estructura para brindar atención adecuada a esta patología, ya sea por falta de estructura, material o falta de información. Centros que soportan la técnica FETO. Se espera que este estudio pueda contribuir al campo del conocimiento de la cirugía pediátrica, allanando el camino para futuras investigaciones.

Palabras clave: Hernias Diafragmáticas Congénitas, Edad Gestacional, Análisis de Supervivencia.

\section{INTRODUÇÃO}

A hérnia diafragmática congênita (HDC) é um defeito que ocorre entre a quarta e oitava semana de desenvolvimento embrionário e resulta na ausência total ou parcial do músculo diafragmático. Permite a passagem de vísceras abdominais para o tórax levando a compressão do pulmão, impedindo assim seu completo desenvolvimento. A ausência de continuidade do músculo diafragmático prejudica os movimentos respiratórios fetais que são importantes para a maturação pulmonar. Como resultado, verifica-se que os recém-nascidos com HDC apresentam uma combinação de hipoplasia pulmonar grave e hipertensão pulmonar (GREER JJ, 2013; MOREIRA MEL, et al., 2004; SANTOS E e RIBEIRO S, 2008).

A incidência geral de defeitos congênitos no Brasil é de 2 a $5 \%$ dos recém-nascidos. A hérnia diafragmática afeta entre 1: 2.000 e 1: 4.000 nascidos vivos, constituindo umas das principais anomalias congênitas (GALLINDO RM, et al., 2015). No período de 2001 a 2009 foram registrado no Departamento de Informática do Sistema Única de Saúde (DATASUS) 1.075 nascimentos com HDC, que corresponde a uma incidência de 1: 25.000 neste período e no estado de São Paulo responde por 1 caso para 14 mil habitantes. Segundo a Organização Mundial de Saúde (OMS) há uma prevalência maior no sexo masculino, mas não há diferença entre raças. É mais frequente à esquerda (80\%) e em $30 \%$ dos casos se associa a anomalias cromossômicas, como a trissomia do 13 ou do 18 (GALLINDO RM, et al., 2015; DOYLE NM e LALLY KP, 2004).

A gravidade da HDC está associada à extensão das vísceras herniadas, bem como o local em que ocorreu a herniação. $O$ aparecimento precoce da doença e a presença do fígado na cavidade torácica são indicativos de mau prognóstico (SANTOS E e RIBEIRO S, 2008; HOROVITZ DDG, et al., 2005; GALLINDO RM, et al., 2015).

O head-lung rate (LHR), conhecido como índice pulmão-cabeça é o melhor método para determinar o grau de hipoplasia pulmonar e consiste na relação entre a superfície do pulmão contralateral à lesão e a circunferência da cabeça em milímetros. É determinado por ressonância magnética e valores abaixo de 1,0 se correlacionam com prognóstico muito ruim, com taxas entre 0 a $20 \%$ de sobrevivência; pacientes com LHR entre 1,0 e 1,4 têm prognóstico intermediário e LHR maior que 1,4 têm bom prognóstico (GALLINDO RM, et al., 2015).

Observou-se que em 95\% dos natimortos com HDC apresentavam outros defeitos associados à hipoplasia pulmonar que contribuem consideravelmente para a mortalidade. Dentre as anomalias congênitas associadas, verificam-se as cardíacas, as urogenitais, gastrointestinais, esqueléticas e do sistema nervoso central (SNC) (MOREIRA MEL, et al., 2004; HARRISON MR, et al., 2003). 
O diagnóstico da HDC pode ser facilmente estabelecido no pré-natal por meio da ultrassonografia (US) (MOREIRA MEL, et al., 2004). Já em caso de HDC com herniação do fígado para a cavidade torácica, podese ter dificuldade em diagnosticá-la em virtude da semelhança na ecogenicidade entre o fígado e o pulmão. Para isso, utiliza-se o Doppler a fim de verificar a presença da veia porta ao nível ou acima do diafragma. A ressonância magnética elucida o caso e determina prognóstico através da medida do LHR (SANTOS E e RIBEIRO S, 2008).

Em 2004, um grupo europeu propôs uma nova técnica para o tratamento da HDC isolada, denominado como Oclusão Endotraqueal Fetoscópica (FETO). Esse procedimento é realizado entre $26^{\circ}$ e $28^{\circ}$ semanas do desenvolvimento embrionário, ao final da fase canalicular, e consiste na colocação de um balão ou PLUG na traqueia fetal a fim bloquear temporariamente a saída do surfactante para a cavidade amniótica, permitindo assim o desenvolvimento pulmonar, maturação da vasculatura, a diminuição da hipoplasia pulmonar e a redução gradual das vísceras abdominais. Esse tratamento é indicado para casos de HDC isolada, cariótipo normal, diagnóstico antes da 25a semana de gestação, fígado herniado e LHR $<1,0$ ou relação LHR observado/esperado (o/e LHR) < 25 (SOUZA JCK, et al., 2018; GALLINDO RM, et al., 2015; PERALTA CFA e BARINI R, 2011; DEPREST J, et al., 2004; JUNIOR FA, et al., 2016; SOUZA JCK, et al., 2018).

O uso da técnica FETO aumentou a taxa de sobrevida de 24,1 para $49,1 \%$ para os casos de HDC a esquerda e de 0 para $35,3 \%$ em $\mathrm{CDH}$ no lado direito. No entanto, as complicações relacionadas ao procedimento como ruptura prematura da membrana e parto prematuro foram consideráveis. Deste trabalho para cá, muitos outros estudos foram realizados, possibilitando assim o aperfeiçoamento dessa técnica e da indicação do procedimento, no entanto, ainda hoje, não se chegou a uma conclusão em relação à eficácia da técnica FETO como melhor tratamento para HDC (JUNIOR FA, et al., 2016).

Frente as dúvidas persistentes sobre a utilização ou não da técnica FETO e a observação de casos mais graves e com necessidade de patch para correção cirúrgica nos pacientes submetidos a técnica, o objetivo deste trabalho foi compreender, por meio da revisão da literatura, a relação entre a escolha da técnica FETO e a sobrevida dos recém-nascidos.

\section{MÉTODOS}

Trata-se de uma revisão integrativa, nos meses de maio a outubro de 2018, cujas bases pesquisadas foram: MEDLINE (Medical Literature Analysis and Retrieval System Online /PubMed) e LILACS (Literatura Latino America e do Caribe em Ciências da Saúde). Os descritores selecionados a partir da terminologia em saúde consultada no Medical Subject Headings Section (MeSH) do PUBMED/MEDLINE foram:"Congenital Diaphragmatic hernia", "Surgical treatment", "Surgical procedure" e "Operative" foram associados ao descritos FETO.

Foram consideradas as publicações dos últimos 06 anos, ou seja, de 2012 a 2018 e apenas estudos realizados em humanos, para análise qualitativa. Após a leitura dos resumos, segundo pertinência e consistência do conteúdo, foram observados os seguintes critérios de inclusão: estudos que relatam desfechos perinatais de gestações com HDC, publicações originais nas línguas portuguesa, espanhola e inglesa; que adotavam uma abordagem qualitativa, considerando o objetivo do estudo. Quanto aos critérios de exclusão: artigos repetidos, resenhas, anais de congresso, artigos de opinião, artigos de reflexão, editoriais, artigos que não abordaram diretamente o tema deste estudo e artigos publicados fora do período de análise. Os resultados encontrados foram triados a partir da leitura do título e resumo. A amostra resultante foi definida através da leitura completa dos artigos.

\section{RESULTADOS E DISCUSSÃO}

Identificaram-se 687 artigos nas bases de dados PubMed e LILACS, constatando-se que, após a aplicação dos critérios de inclusão/exclusão e a leitura dos títulos e resumos, 145 não possuíam aderência à temática, restando 12 artigos para inclusão na revisão. A Figura 1 apresenta a estratégia de busca dos artigos nas bases de dados. 
Figura 1 - Fluxograma do processo de seleção do estudo para a revisão integrativa, Parnaíba-PI, 2018.

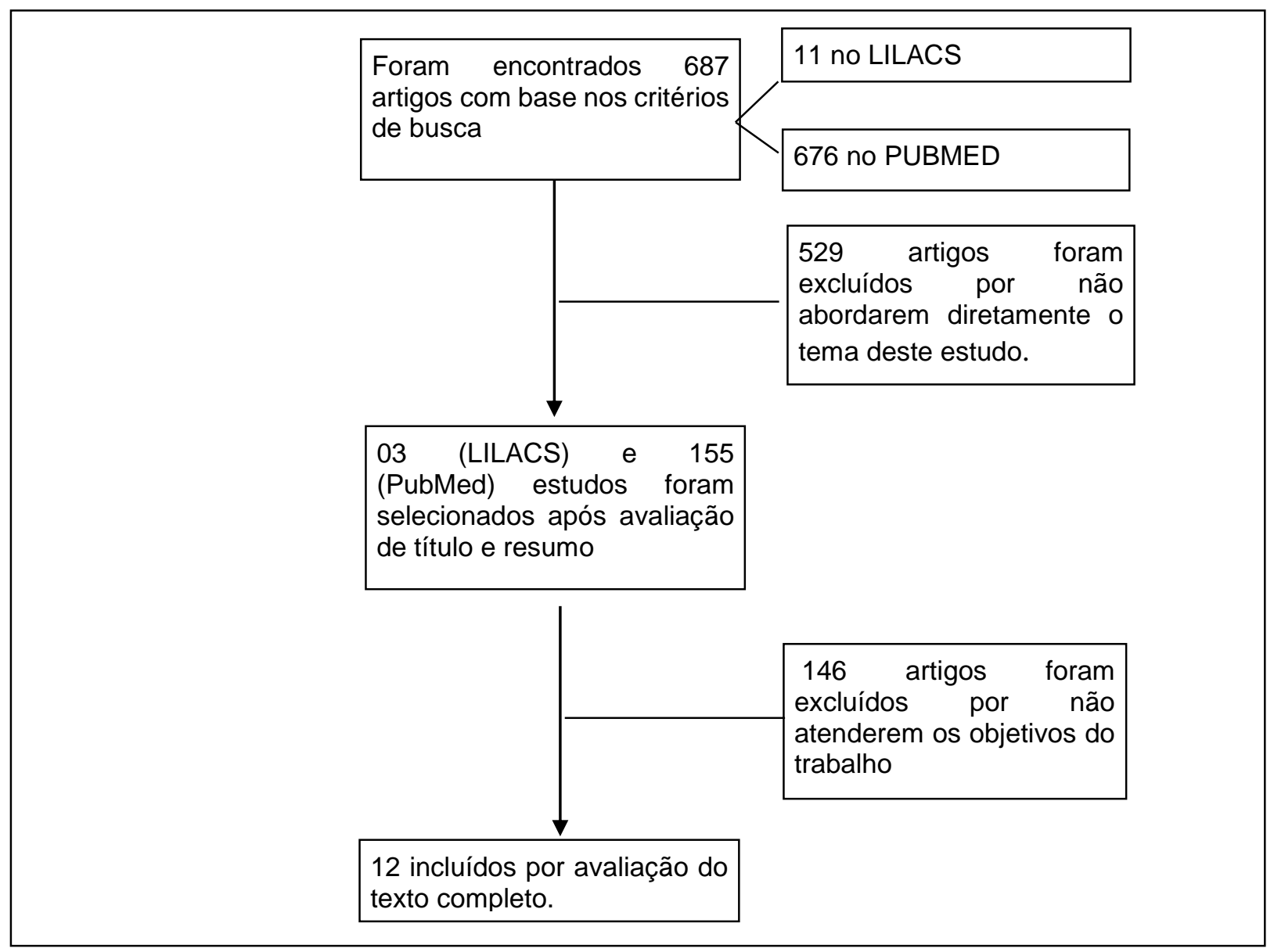

Fonte: Dados de pesquisa, 2019.

A hérnia diafragmática congênita (HDC) é um defeito embriológico que leva a ausência parcial ou total do músculo diafragmático, ocasionando a herniação das vísceras abdominais, o que prejudica o desenvolvimento completo do pulmão. A incidência no Brasil varia de 2 a $5 \%$ dos recém-nascidos e afetam 1: 2.000 e 1: 4.000 nascidos vivos, constituindo umas das principais anomalias congênitas. Habitualmente apresenta alta taxa de mortalidade devido ao grave comprometimento respiratório e cardíaco.

Nos últimos 30 anos, a HDC foi considerada como uma síndrome, em que incluiu hipoplasia pulmonar, imaturidade pulmonar, hipoplasia do lado esquerdo do coração e hipertensão pulmonar persistente neonatal. Dessa forma, a HDC deve ser considerada uma emergência cirúrgica (GALLINDO RM, et al., 2015; AIHOLE JS, et al., 2018).

A HDC pode ser diagnosticada por ultrassonografia durante consulta de pré-natal, a partir da $14^{\circ}$ ou $15^{\circ}$ semana de gestação. Segundo a literatura, nas últimas três décadas, as taxas de diagnóstico pré-natal melhoraram significativamente de $15 \%$ meados dos anos 80 a $83 \%$ nos anos 2010 , sendo a maioria detectada após 24 semanas de gestação.

Este aumento se deve em virtude de uma combinação entre aperfeiçoamento dos equipamentos de US e melhor conhecimento das características de US da HDC, tais como presença do estômago cheio de líquido dentro da cavidade torácica e desvio do lado direito do mediastino.

Em atenção ao objetivo desta pesquisa, elaborou-se uma síntese dos principais desfechos dos doze artigos incluídos na revisão, tendo como intenção analisar se o tempo de diagnóstico da patologia interfere na elegibilidade para a técnica (Quadro 1). 
Quadro 1 - Artigos selecionados durante a coleta de dados da revisão integrativa da literatura. Parnaíba, 2019.

\section{№ AUTOR}

1 Toro $\mathrm{MNH}$, et al
ANO

2012

Descrever o manejo e os resultados dos pacientes com Hérnia Diafragmática Congênita entre 1999 e 2009,em um hospital pediátrico, em Medellín, Colômbia.

2 Aihole JS, et 2018 Analisar o perfil clínico e o resultado da Hérnia Diafragmática al referência neonatal e pediátrico em Karnataka, Índia.

3 Garriboli M, 2012 Avaliar se o tratamento e a sobrevivência mudaram durante et al a última década.

4 Ruano R, et 2012 Determinar se a técnica de oclusão traqueal endoscópica al fetal (FETO) melhora a sobrevida em casos de Hérnia Diafragmática Congênita isolada grave.

5 Burgos MC, 2016 Comparar os desfechos em gestações com detecção préet al natal de Hérnia Diafragmática Congênita (HDC) em crianças diagnosticadas após o nascimento, tratadas na mesma instituição e determinar a capacidade de prever o prognóstico por meio da medida da relação pulmão-a-cabeça observada e esperada (O / E LHR).

\section{CONCLUSAOO}

No Hospital de Medellin, 45 pacientes diagnosticados com hérnia diafragmática congênita foram tratados entre 1999 e 2009; este estudo incluiu 36 deles, dos quais 10 morreram (27,8\%); somente em um paciente a morte foi associada ao procedimento cirúrgico. Os fatores associados à mortalidade foram instabilidade hemodinâmica no momento da avaliação, Apgar menor que 5, uso de adesivo para correção cirúrgica, tratamento exclusivamente médico, diagnóstico pré-natal e presença de malformações associadas.

Hérnia Diafragmática Congênita são comuns no lado esquerdo com prognóstico razoavelmente bom. No entanto, os HDC do lado direito são raros; eles carregam um bom prognóstico.

Esta análise multicêntrica indica que a taxa de sobrevivência de crianças com Hérnia Diafragmática Congênita, referenciada em dois centros cirúrgicos pediátricos europeus, é alta (79\%). Observaram um aumento significativo na proporção de bebês submetidos à cirurgia, mas isso não resultou em um aumento significativo na taxa de sobrevida global.

A técnica FETO melhora a sobrevivência neonatal em casos com HDC grave isolada.

Crianças com hérnia diafragmática congênita diagnosticada no período pré-natal representam uma população com uma condição mais grave em comparação com crianças diagnosticadas após o nascimento. Eles têm desfechos mais pobres com maiores necessidades de Oxigenação por membrana extracorpórea ou uso de adesivo, e menores taxas de sobrevida foram observadas na relação pulmão-a-cabeça observada e esperada abaixo de $35 \%$. 
6 Dues JW, et 2015 Analisar características e resultados da Hérnia Diafragmática al Congênita a direita

7 Botden SM, 2017 Apresenta os resultados pacientes com Hérnia Diafragmática et al Congênita bilateral.
Pré-natal o diagnóstico e o reparo do adesivo foram associados a um aumento da taxa de mortalidade em hérnia diafragmática congênita direita. No entanto, a morbidade após a reparação da hérnia diafragmática congênita a direita não foi significativamente diferente de que em HDC a esquerda em sobreviventes.

O tratamento de pacientes com HDC bilateral permanece desafiador com uma alta taxa de mortalidade. Baixos índices de Apgar, Oxigenação por membrana extracorpórea (provavelmente como um substituto para a gravidade da doença) e reparo do patch foram negativamente associados com o desfecho.

\begin{tabular}{|c|c|c|c|c|}
\hline 8 & $\begin{array}{l}\text { Bouchghoul } \\
\mathrm{H} \text {, et al }\end{array}$ & 2015 & $\begin{array}{l}\text { Estudar a relação entre idade gestacional no diagnóstico e } \\
\text { mortalidade e morbidade em fetos com hérnia diafragmática } \\
\text { congênita isolada. }\end{array}$ & $\begin{array}{l}\text { A idade gestacional no momento do diagnóstico é um preditor } \\
\text { independente de prognóstico pós-natal para crianças que apresentam } \\
\text { hérnia diafragmática congênita isolada e deve ser levada em } \\
\text { consideração na estimativa da morbidade e mortalidade pós-natal. }\end{array}$ \\
\hline 9 & Ali K et al & 2013 & $\begin{array}{l}\text { Avaliar a mortalidade e morbidade de lactentes com hérnia } \\
\text { diafragmática congênita que foram submetidos a oclusão } \\
\text { traqueal endoscópica fetal (FETO) e se isso foi influenciado } \\
\text { pelo nascimento prematuro. }\end{array}$ & $\begin{array}{l}\text { Os resultados enfatizam a necessidade de reduzir o parto prematuro } \\
\text { após FETO. }\end{array}$ \\
\hline 10 & Ali K et al & 2016 & $\begin{array}{l}\text { Testar a hipótese de que os bebês com Hérnia Diafragmática } \\
\text { Congênita que haviam se submetido a técnica FETO, em } \\
\text { comparação àqueles que não o tinham, teriam taxas de } \\
\text { sobrevida semelhantes, mas que apresentariam maior } \\
\text { morbidade. }\end{array}$ & $\begin{array}{l}\text { O grupo FETO aumentou a morbidade, mas não a mortalidade. O } \\
\text { menor índice de oxigenação nas primeiras } 24 \text { horas foi o melhor } \\
\text { preditor de sobrevivência, independentemente da intervenção pré- } \\
\text { natal }\end{array}$ \\
\hline 11 & $\begin{array}{l}\text { Snoek KG et } \\
\text { al }\end{array}$ & 2018 & $\begin{array}{l}\text { O objetivo deste estudo foi determinar se houve tendências } \\
\text { na sobrevida na última década e comparar populações de } \\
\text { pacientes, opções de tratamento e taxas de sobrevivência } \\
\text { entre } 4 \text { centros de alto volume e, portanto, determinar quais } \\
\text { fatores estavam associados à sobrevida. }\end{array}$ & $\begin{array}{l}\text { As populações de pacientes eram diferentes entre os centros, o que } \\
\text { influenciou os resultados. Houve uma variabilidade significativa na } \\
\text { sobrevida ao longo do tempo e entre os centros. }\end{array}$ \\
\hline 12 & $\begin{array}{l}\text { Sananes } N \text {, } \\
\text { et al }\end{array}$ & 2016 & $\begin{array}{l}\text { Avaliar a associação independente entre a resposta pulmonar } \\
\text { fetal e a prematuridade aos desfechos pós-natais após } \\
\text { oclusão traqueal fetal para hérnia diafragmática congênita. }\end{array}$ & $\begin{array}{l}\text { A resposta pulmonar fetal após FETO é o fator mais importante } \\
\text { associado à sobrevida, independentemente da idade gestacional no } \\
\text { momento do parto. }\end{array}$ \\
\hline
\end{tabular}

Fonte: Dados da pesquisa, 2019. 
Observou-se que a maioria dos diagnósticos foram realizados durante o pré-natal, exceto em no estudo de coorte de Aihole JS et al. (2018) e o estudo de Garriboli M et al.(2012) em que constaram um maior diagnóstico durante o pós-natal e isso pode ser justificado por conta da rara herniação intermitente das vísceras, tamanho do defeito ou falha no acompanhamento durante o pré-natal (MOREIRA MEL, et al., 2004; SOUZA JCK, et al., 2018; SANTOS E e RIBEIRO S, 2008; LEE HS, et al., 2018 ; BURGOS CM, et al., 2018).

Nestes casos, a detecção ao nascimento dá-se por sinais de sofrimento respiratório (taquipneia, retrações, cianose), abdome escavado, assimetria torácica, dextrocardia e murmúrio vesicular abolido ou diminuído. Em relação ao diagnóstico, a localização do defeito a esquerda é mais frequentemente detectada quando comparados a HDC a direita, e isso resulta tanto por conta da incidência, quanto por causa da dificuldade de diagnóstico (MOREIRA MEL, et al., 2004 ; SOUZA JCK, et al., 2018; KALANJ J, et al., 2016; SANTOS E e RIBEIRO S, 2008; BURGOS CM, et al., ,2018; DUES JW, et al.,2015).

Em relação à mortalidade, verificou-se que ainda hoje, apesar de um maior conhecimento da história natural da doença e dos avanços no tratamento clínico e cirúrgico, o diagnóstico pré-natal precoce está associado a um pior prognóstico em comparação com o diagnóstico pós-natal da mesma condição. Os estudos de Toro MNH et al. (2012), Burgos CM et al.(2016), Botden SM et al. (2017) e Snoek KG et al.(2018) referem que a mortalidade é maior em pacientes com diagnóstico pré-natal quando comparado ao grupo de diagnóstico pós-natal.

Pressupõe-se que a HDC diagnosticada precocemente seja caracterizada por um defeito maior no diafragma, mais evidentes no exame pré-natal, com maior conteúdo visceral no tórax, o que resulta em um efeito de massa dos órgãos herniados, levando a graus variáveis de hipoplasia pulmonar e à hipertensão pulmonar persistente. Segundo o artigo de Bouchghoul $\mathrm{H}$ et al. (2015), existe uma relação significativa entre a idade de diagnóstico gestacional na HDC e morbidade e mortalidade (BURGOS CM, et al., 2016; BURGOS CM, et al., 2019; RUANO R, et al., 2009; AlHOLE JS, et al.,2018; BOUCHGHOUL H, et al., 2015).

Esses resultados devem ser analisados com ressalvas, pois se observa um número significativo de óbitos fetais e neonatais que não são reconhecidos ou que ocorrem antes da transferência para um centro de atendimento terciário, sobretudo em países em desenvolvimento onde existem poucos centros especializados nesse tipo de tratamento. Por conseguinte, os pacientes que são atendidos nos grandes centros representam uma pequena parcela dos casos de HDC.

Harrison MR et al. (2003) descreveu essa disparidade como "mortalidade oculta" da HDC e isso explica parcialmente porque neonatos com diagnóstico pós-natal apresentam melhores taxas de sobrevivência e defeitos diafragmáticos menores. Além disso, em países onde o aborto é legalizado, o diagnóstico de HDC durante o pré-natal pode configurar-se como uma "mortalidade oculta", já que tamanhos maiores de defeitos diagnosticados no período pré-natal podem optar pela interrupção da gravidez.

Um estudo retrospectivo realizado na Austrália verificou que houve um aumento de cerca de $30,7 \%$ na taxa de aborto após o diagnóstico pré-natal de HDC (BURGOS CM, et al., ,2018; AlHOLE JS, et al.,2018; BURGOS CM, et al., 2016; HARRISON MR, et al., 2003; LEE HS, et al., 2018; BOUCHGHOUL H, et al., 2015).

Além da idade gestacional ao diagnóstico, os seguintes fatores estão relacionados com mal prognóstico: apresentação de sintomas nas primeiras seis horas de vida, Apgar menor que 5 no quinto minuto, baixo peso ao nascer e associações de malformações, bem como a instabilidade hemodinâmica na avaliação pelo cirurgião, uso de prótese para o reparo da HDC, hipoplasia pulmonar e hipertensão primária pulmonar (TORO MNH, et al., 2012; AlHOLE JS, et al., 2018).

O tratamento cirúrgio durante o pré-natal é feito por meio da técnica de oclusão traqueal fetal endoscópica que é um procedimento minimamente invasivo e foi desenvolvido como uma alternativa para propiciar 0 crescimento do pulmão fetal. Estudos apontam que a técnica FETO é única opção de tratamento clinicamente disponível que pode reduzir hipoplasia pulmonar e oferecem crescimento pulmonar durante a gestação se a condição for identificada e devidamente avaliada. 
As indicações para esse procediemento são hérnia diafragmática isolada, cariótipo normal, diagnóstico antes da $25^{\text {a }}$ semana de gestação, LHR $<1,0$ ou relação O/E LHR $<25$. Em nossa revisão foi observado um aumento do risco significativo de prematuridade, bem como ruptura prematura de membrana (SILVEIRA SLA, et al., 2015; ROHDE L, et al., 2018; RUANO R, et al., 2012; ALI K, et al., 2016; ALI K, et al., 2013; BURGOS CM, et al.,2018).

Durante nosso estudo, a cerca dessa técnica, encontramos que os estudos de Snoek KG et al. (2018), apontam que a FETO não foi associada à mortalidade, mas ele foi usado de forma mais significativa entre 0 grupo não sobrevivente, refletindo um critério de seleção para uso mais precavido. Entretanto, o estudo de Ruano $\mathrm{R}$ et al. (2012), relata que os pacientes com HDC isolada grave submetidos a FETO tiveram melhor sobrevida em comparação com casos que receberam apenas cuidados intensivos pós-natais ( $50 \%$ vs $4,8 \%$ ).

Tal estudo é reafirmado por um estudo de coorte desenvolvido na Europa, que relata uma taxa de $50 \%$ de sobrevida nos pacientes submetidos a FETO. No entanto, esses estudos que relatam sobre um aumento na sobrevida devem ser interpretados com cautela, pois, podem apresentar enviesamento na seleção da população, impedindo qualquer conclusão no que diz respeito à sua influência na sobrevivência. Além disso, sabe-se que o parto realizado fora de um centro perinatal aumenta a mortalidade em recém-nascidos com HDC. Ademais, observa-se uma baixa disponibilidade ao acesso da técnica FETO em muitos centros de tratamento (GREER JJ, et al., 2013; BURGOS CM, et al. ,2018; RUANO R, et al., 2012; SNOEK KG, et al., 2018; DEPREST J, et al., 2004).

De acordo com a análise retrospectiva de Ali K et al. (2013), entre o grupo que havia submetido a FETO, os nascidos antes da $35^{\circ}$ semanas estavam relacionados diretamente com a maior mortalidade quando comparados aos que haviam nascidos a termo. Entretanto, não houve pacientes que conseguiram resistir antes das 33 semanas. Os prematuros tiveram LHR menor, consequentemente, tiveram uma hipoplasia pulmonar mais grave que pode ter contribuido com a mortalidade. No entanto, Sananes $\mathrm{N}$ et al. (2016) em seu estudo revela que a sobrevida e a resposta pulmonar em pacientes que realizaram FETO, são independentes da idade gestacional do parto e que a prematuridade e não está correlacionada com esse desfecho, havendo 4 sobreviventes de 16 fetos nascidos antes da 32 semanas.

Em relação ao tratamento clínico, a literatura aponta que os avanços nas unidades de terapia intensiva, como a utilização de VOAF (Ventilação Oscilatória De Alta Frequência), o uso de NOi e o uso de ECMO melhoraram o prognóstico do paciente com HDC em estado grave. Estudos recentes mostraram melhora da sobrevida para $\mathrm{CDH}$ isolada de $85 \%$ a $90 \%$, o que envolve a implementação de protocolos como métodos agressivos de controle da hipertensão pulmonar, grande apoio monetário e disponibilidade de ECMO.

Entretanto, os benefícios dessas várias técnicas e fármacos ainda estão sendo debatidos, e até agora não há concordância geral nas condutas adotadas nos diversos serviços de neonatologia e cirurgia pediátrica. Observou-se nesta revisão que a maioria do tratamento médico foi realizado principalmente com VOAF, ECMO, NOi e inotrópicos (dopamina e/ou dobutamina). No entanto, verificou-se que os pacientes que realizaram apenas o tratamento clínico apresentaram uma alta mortalidade (ROHDE L, et al., 2018; DAVIS C et al; AlHOLE JS, et al.,2018; TORO MNH, et al., 2012; BURGOS CM, et al., 2016).

Com relação ao tempo para realizar a cirúrgia de reparo, não há divergências na literatura, pois para proceder a cirúrgia, o neonanto com HDC deve estar fisiologicamente estável. No entanto, por décadas acreditavam-se que o reparo imediato após o nascimento, com a retirada do conteúdo abdominal da cavidade torácica, diminuiria a comprensão dos pulmões e consequentemente aumentaria as taxas de sobrevidas.

Com isso, após uma melhor compreensão da fisiopatologia da HDC, revelou que os neonatos que foram subemetidos a cirúrgia no primeiro dia de vida passavam por breve perído de lua de mel definido com trocas gasosas apropriados, subsequentes por declínio respiratório progressivo, hipertensão pulmonar shunting direito para a esquerda, hipoxemia e, por conseguinte morte por insuficiência respiratória. Nesta revisão encontrou-se que dos pacientes submetidos ao tratamento cirúrgico pós-natal, o reparo primário foi mais frequente em pacientes que o defeito diafragmático era maior (ZANI A, et al., 2014; AlHOLE JS, et al.,2018; TORO MNH, et al., 2012; GARRIBOLI M, et al.,2012). 
Em relação ao tratamento cirúrgico, os neonatos com HDC podem ser reparados por várias abordagens cirúrgicas, como as técnicas abertas transabdominais ou transtóracicas, assim como por técnicas minimamente invasivas laparoscópicas ou toracoscópicas. Entretanto, nenhuma dessas abordagens cirúrgicas apresentam supremacia absoluta (ZANI A, et al., 2014).

Apesar dos recentes avanços na terapia clínico-cirúrgica em pacientes com HDC, não se conseguiu obter uma melhoria da sobrevida. Segundo os estudos de Fumino $S$ et al. (2005) a taxa de mortalidade está entre $25 \%$ e 55\%, devido, sobretudo, à hipoplasia pulmonar e a hipertensão pulmonar persistente. Em contrapartida, no estudo multicentrico de Garriboli $\mathrm{M}$ et al, a taxa de sobrevivência de crianças com HDC foi de $79 \%$, observando-se um aumento significativo na proporção de bebês submetidos à cirurgia, mas isso não resultou em um aumento significativo na taxa de sobrevida global (AIHOLE JS, et al.,2018; TORO MNH, et al., 2012; RUANO R, et al., 2012; FUMINO S, et al., 2005; GARRIBOLI M, et al., 2012).

\section{CONSIDERAÇÕES FINAIS}

Nos últimos seis anos houve avanços no conhecimento de fatores dos prognósticos, no tratamento clínico pré e pós-natal, no domínio de técnicas de intervenção intrauterina e consequentemente melhora nos resultados após realização de FETO. Porém a patologia exige muitos recursos tecnológicos e cujos esforços são pouco recompensados nos casos mais graves. Uma melhora na notificação e detecção da doença pode levar estes casos a centros mais equipados para otimizar o estudo e cuidados do binômio gestante-feto, e assim avançar ainda mais no tratamento da doença. A HDC diagnosticada durante o pre-natal permanece um desafio, pois casos mais graves são detectados precocemente e e nem todo os serviços são capazes de oferecer o tratamento adequado para esta patologia, seja por falta de estrutura, material ou por ausência de centros que realizem abordagens coadjuvantes como a FETO. Assim, com esse estudo espera-se contribuir para o campo de conhecimento da cirurgia pediátrica, abrindo caminho para novas pesquisas que relacionam a idade do diagnóstico gestacional com o uso da técnica FETO e com a elegibilidade do tipo de reparo pósnatal. Sugere-se que mais estudos multicentricos devem ser feitos a fim de elucidar melhor os reais benefícios da técnica FETO.

\section{REFERENCIAS}

1. AIHOLE JS, et al. A Clinical Study on Congenital Diaphragmatic Hernia in Neonates: Our Institutional Experience. J Indian Assoc Pediatr Surg. 2018 Jul-Sep; 23(3):131-139.

2. ALI K, et al. Outcome of CDH infants following fetoscopic tracheal occlusion - influence of premature delivery. J Pediatr Surg. 2013 Sep; 48(9):1831-6.

3. ALI K, et al. Congenital diaphragmatic hernia-influence of fetoscopic tracheal occlusion on outcomes and predictors of survival. Eur J Pediatric. 2016 Aug; 175(8):1071-6.

4. BOTDEN SM, et al. Bilateral congenital diaphragmatic hernia: prognostic evaluation of a large international cohort. J Pediatr Surg. 2017 Sep; 52(9):1475-1479.

5. BOUCHGHOUL $\mathrm{H}$, et al. Congenital diaphragmatic hernia: does gestational age at diagnosis matter when evaluating morbidity and mortality? American Journal of Obstetrics and Gynecology, 213(4), 535.e1-535.e7.

6. BRASIL. Ministério da Saúde. Conselho Nacional de Saúde. 2012. Resolução CNS no 466 de 12 de dezembro de 2012.

7. BURGOS CM, et al. Hérnia diafragmática congênita pré-natal versus pós-natal - Lado, estágio e desfecho. Jornal de Cirurgia Pediátrica 2018. doi: 10.1016 / j.jpedsurg.2018.04.008

8. BURGOS CM et al. Differences in Outcomes in Prenatally Diagnosed Congenital Diaphragmatic Hernia Compared to Postnatal Detection: A Single-Center Experience. Fetal Diagn Ther. 2016;39(4):241-7.

9. DAVIS C e WALKER GM. Desafio de determinar o verdadeiro resultado da hérnia diafragmática congênita. Arquivos de Doenças na Infância - Edição fetal e neonatal, fetalneonatal - 2018-314820. doi: 10.1136 / archdischild-2018-314820

10. DEPREST J, et al. Fetoscopic tracheal occlusion (FETO) for severe congenital diaphragmatic hernia: evolution of a technique and preliminary results. Ultrasound in Obstetrics and Gynecology 2004;24(2):121-6.

11. DIFIORE JW, et al. Experimental fetal tracheal ligation reverses the structural and physiological effects of pulmonary hypoplasia in congenital diaphragmatic hernia. Journal of Pediatric Surgery 1994;29(2):248-56. 
12. DOYLE NM e LALLY KP. The CDH Study Group and advances in the clinical care of the patient with congenital diaphragmatic hernia.Semin Perinatol. 2004;28(3):174-84

13. DUESS JW et al. Outcome of right-sided diaphragmatic hernia repair: a multicentre study. Pediatr Surg Int. 2015 May;31(5):465-71.

14. FUMINO S, et al. A clinical analysis of prognostic parameters of survival in children with congenital diaphragmatic hernia. Eur J Pediatr Surg. 2005 Dec;15(6): 399 - 403.

15. GALLINDO RM, et al. Manejo pré-natal da hérnia diafragmática congênita: presente, passado e futuro, Rev. Bras. Ginecol. Obstet. vol.37 no.3 Rio de Janeiro Mar. 2015

16. GARRIBOLI M, et al. Trends in the treatment and outcome of congenital diaphragmatic hernia over the last decade. Pediatr Surg Int. 2012 Dec;28(12):1177-81.

17. GREER JJ. Current concepts on the pathogenesis and etiology of congenital diaphragmatic hérnia. Respiratory Physiology \& Neurobiology 189 (2013) 232-240.

18. GRIVELL RM, et al. Prenatal interventions for congenital diaphragmatic hernia for improving outcomes (Review). Cochrane Database of Systematic Reviews, Ed. 11. 2015.

19. HARRISON MR, et al. Fetoscopic temporary tracheal occlusion for congenital diaphragmatic hernia: prelude to a randomized, controlled trial. J PediatrSurg 2003;38(7):1012-1020

20. HOROVITZ DDG, et al. Atenção aos defeitos congênitos no Brasil: panorama atual. Cad. Saúde Pública, Rio de Janeiro, 21(4):1055-1064, jul-ago, 2005

21. JUNIOR EA, et al. Procedure-Related Complications and Survival Following Fetoscopic Endotracheal Occlusion (FETO) for Severe Congenital Diaphragmatic Hernia:Systematic Review and Meta-Analysis in the FETO Era. Eur J Pediatr Surg. 2016.

22. KALANJ J, et al. Congenital diaphragmatic hernia - a Belgrade single center experience. J Perinat Med. 2016 Oct $1 ; 44(8): 913-8$.

23. LAUDY JAM, et al. Congenital diaphragmatic hernia: an evaluation of the prognostic value of the lung-to-head ratio and other prenatal parameters. Prenat Diagn 2003;23: 634-9.

24. LEE H, et al. Hérnia diafragmática congênita: impacto das estratégias contemporâneas de manejo nos resultados perinatais. American Journal of Obstetrics and Gynecology 2018. doi: 10.1002 / pd.5376

25. METKUS AP, et al. Sonographic predictors of survival in fetal diaphragmatic hernia. J Pediatr Surg 1996;31:14851; discussion 151-2.

26. MOORE KL, et al. Embriologia clínica. 8a ed. Rio de Janeiro: Elsevier; 2008.

27. MOREIRA MEL, et al. O recém-nascido de alto risco: teoria e prática do cuidar. Rio de Janeiro: Editora FIOCRUZ, 2004. 564 p.

28. PERALtA CFA e BARINI R. Cirurgia fetal no Brasil. Rev. Bras. Ginecol. Obstet. vol.33 no.4 Rio de Janeiro Apr. 2011

29. ROHDE L, et al. Rotinas em cirurgia digestiva. 3 ed. Grupo A - Artmed; 2018. Editado como livro impresso em 2018. ISBN 978-85-8271-471-3

30. RUANO R, et al. Prediction and probability of neonatal outcome in isolated congenital diaphragmatic hernia using multiple ultrasound parameters. Ultrasound Obstet Gynecol 2012;39:42-9

31. SANANES N, et al. Prematurity and fetal lung response after tracheal occlusion in fetuses with severe congenital diaphragmatic hernia. J Matern Fetal Neonatal Med. 2016 Sep;29(18):3030-4

32. SANTOS E e RIBEIRO S. Hérnia diafragmática congénita - artigo de revisão. Acta ObstetGinecol, Portugal, v. 2, n. 1, p. 25-33, 200.mar. 2008.

33. SILVEIRA SLA, et al. Oclusão traqueal fetal endoscópica (FETO) na Hérnia Diafragmática Congênita grave à direita. 2015. 9 f. Dissertação (Mestrado) - Curso de Medicina, Faculdade de Medicina de Botucatu, Meparem, 2015.

34. SOUZA JCK, et al. Defeitos congênitos da parede abdominal e do diafragma. In: ROHDE, L; OSVALDT, A. B. Organizadores. Rotinas em cirurgia digestiva. 3. ed.Porto Alegre. Artmed, 2018. Cap12, p90-107.

35. SNOEK KG, et al. Congenital Diaphragmatic Hernia: 10-Year Evaluation of Survival, Extracorporeal Membrane Oxygenation, and Foetoscopic Endotracheal Occlusion in Four High-Volume Centres. Neonatology. 2018;113(1):63-68.

36. TORO MNH, et al. Hernia diafragmática congénita. Experiencia en el Hospital Universitario San Vicente de Paúl, Medellín, Colombia, 1999-2009. latreia vol.25 no.3 Medellín July/Sept. 2012.

37. ZANI A et al. Advances in the surgical approach to congenital diaphragmatic hernia. Seminars in Fetal and Neonatal Medicine, 19(6), 364-369. 